\title{
Engineering Ethics of Neuralink Brain Computer Interfaces Devices
}

\section{Jawad $A J^{1,2 *}$}

${ }^{1}$ Department of Polymer and Petrochemicals Industrials Engineering, College of Materials Engineering University of Babylon, Iraq

${ }^{2}$ School of Engineering and Materials Science, Queen Mary University of London, UK

*Corresponding author: Akram Jassim Jawad, Department of Polymer and Petrochemicals Industrials Engineering, College of Materials Engineering University of Babylon, Iraq, Tel: 009647804652125; Email: akrammaterials4@gmail.com

\section{Perspective \\ Volume 4 Issue 1}

Received Date: January 30, 2021

Published Date: February 19, 2021

DOI: $10.23880 / a b c a-16000160$

\section{Abstract}

Nowadays, smart home devices have started to take a part in everything in our life, which mainly have been developed to consist from brain computer interface (BCI). In recent months, Neuralink BCI (1024-Electode) has been approved to be used by Food and Drug Administration (FDA) in the USA. That makes the ethical related studies have more attention to apply these devices and technologies in our daily life with more security. In this work, the ethical challenges of smart home systems that use BCI for personal monitoring, such as Neuralink Interfaces, have been reviewed, analysed and discussed regarding the fundamental principles in 'Statement of Ethical Principles for the Engineering Profession' of the UK. Firstly, a brief introduction of Neuralink BCI technology and important applications in daily life were discussed with related ethics issues. Then, proposed solutions and recommendations for every situation have been introduced and discussed as well. The main proposed ways to address that are establishing and introducing the related laws and rules, technology development of security and safety, and educate for acceptance culture in the society.

Keywords: Brain Computer Interface (BCI); Engineering Ethics; Smart Home; Neuralink

\section{Introduction}

One of the most important and recent smart home devices for personal monitoring based on brain computer interface (BCI) is Neuralink BCI implanted that invented and produced by Elon Musk company "Neuralink" [1]. Recently, Neuralink BCI (1024-Electode) has been approved to be used by Food and Drug Administration (FDA) in the USA, which makes the ethical related studies have more attention to apply these devices and technologies in smart home applications in more security [2]. This device can help disability people to communicate better and control devices by them brain thinking activity Collinger JL, et al. [3]. For example, this device can transmit the inner speech activity of the brain into an external speaker device for people who suffer speaking problems and illnesses such as locked-in syndrome LIS [4]. Moreover, this technology could produce a revolution in the treatment of different human brain related illnesses, for example epilepsy, schizophrenia, paralysis, and even brain injury. However, these devices could also make the society have social illness related with ethics issues such as inequalities, hackers, governments or people to manipulate and control other people, and many other social problems. The aim of this report is to discuss and write a personal reflection on the ethical challenges of smart home systems that use BCI for personal monitoring, such as Neuralink Interfaces, regarding the fundamental principles in 'Statement of Ethical Principles for the Engineering 
Profession'. Therefore, a brief introduction of Neuralink BCI technology and important applications in daily life were discussed with related ethics issues, and proposed solutions for every case as well.

\section{Neuralink Brain Computer Interfaces}

Generally, the main components and steps in any BCI process were shown in Figure 1, which includes firstly an implanted array of electrodes to read brain signals, and then transition of that signals information into a receiver machine such as a computer. That BCI has the ability to read brain signals data and apply it in the control process for a specific activity [5]. However, in the implanted process of BCI device from Neuralink Company, about 3072 electrodes per array with 32 electrodes per bundle are inserted by robot. These electrodes give the ability to read and write the brain to allow humans to communicate and control many external devices [6]. Schematics of The implanted device in human, design and an experimental model in a rat model of BCI and a USB-C interface by Neuralink company were shown in Figure 2 a-c respectively Musk E, et al. [7]. Consequently, there are a number of ethical issues that have to take in account when this device used in any application, such as health care or even in entertainment systems.

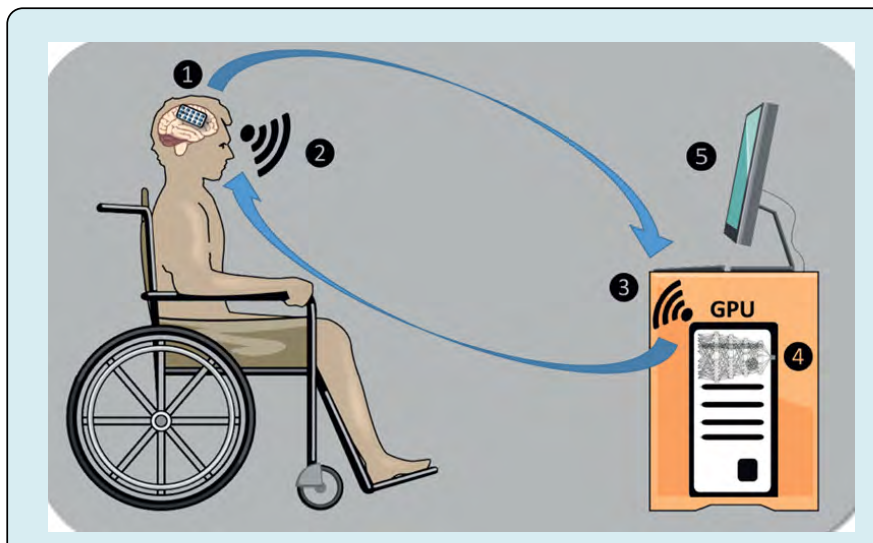

Figure 1: An example Illustration of a brain computer interface product that contain

1. Implanted array of electrodes to read brain signals and

2. Transition of that signals information into

3. A receiver machine such as computer

4. Which has ability to read brain signals data

5. To apply it in control process for a specific activity [5].

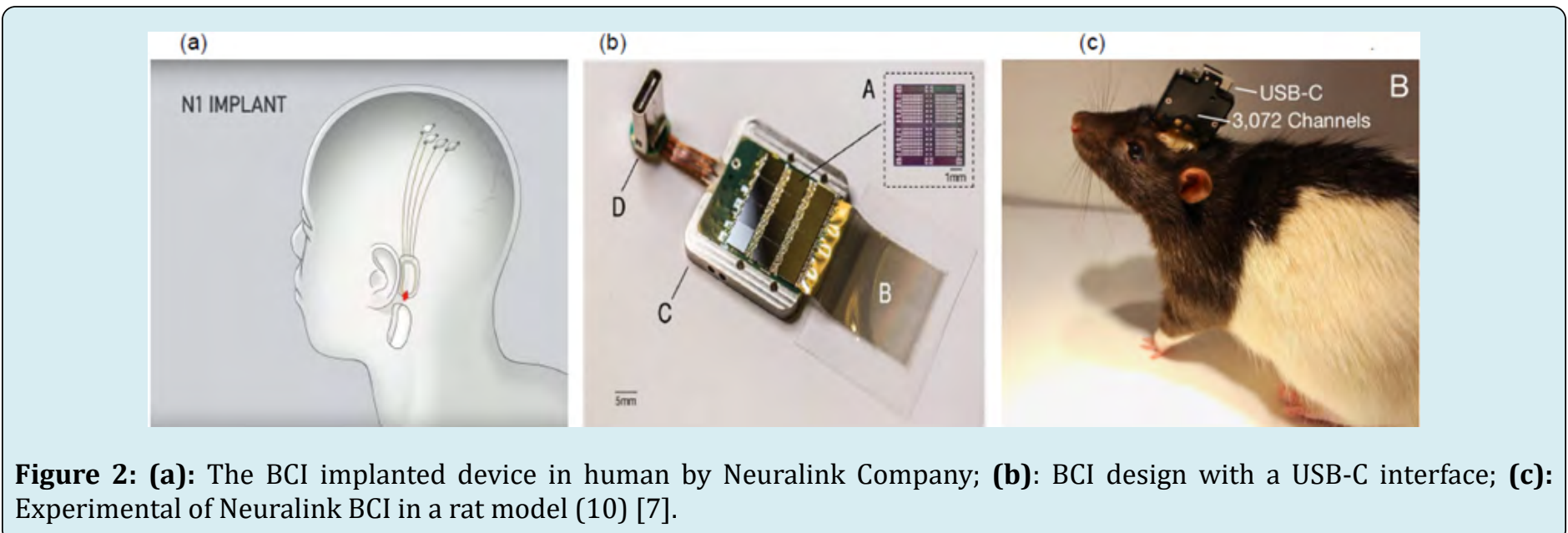

\section{Ethical Issues}

One of the most related fundamental principles to Neuralink BCI technologies and devices in 'Statement of Ethical Principles for the Engineering Profession' is point two, which titled "Respect for life, law, the environment and public good". Their main points were summarized in Table 1 with clarify the comment on the application possibility of these limits in the targeted devices of this report. In the literature, it has been suggested that there are four different areas of ethics concern, which are privacy, agency, identity, and bias as well [8]. It has been mentioned that the governments have to establish regulations, rules, and laws depending on them public ethnicities, religious and socio-economic culture for them nations. Figure 3 represents a summarizing show which covers all possible ethics issues in BCI that proposed by Coin A, et al. [9], which includes three main factors are physical, physiological and social actors [9]. The physical factor is just the user safety; the physiological factors are humanity and personhood, as well as autonomy. While the social factors are stigma and normality, responsibility and regulation, research ethics and informed consent, privacy and security, and justice Coin A, et al. [9]. 


\begin{tabular}{|c|c|}
\hline $\begin{array}{c}\text { Regulations/Laws (Statement of Ethical } \\
\text { Principles, 2014) }\end{array}$ & Possible Application in BCI products \\
\hline Health and safety requirements & Low, and needs to rewriting and establishing \\
\hline Clarify the hazards & Needs to be more clear and specified \\
\hline Lawful and justified work & $\begin{array}{c}\text { Needs to be acceptable in local or national organization, such as the MHRA } \\
\text { (Medicines and Healthcare products Regulatory Agency) in the UK }\end{array}$ \\
\hline Physical and cyber security & Low, and needs to provide a technology with high safety \\
\hline $\begin{array}{c}\text { Personal information and intellectual } \\
\text { property }\end{array}$ & Needs both establishing laws and invented technology to provide high safety \\
\hline
\end{tabular}

Table 1: The main factors of point 2 in fundamental principles in the UK that were written in 'Statement of Ethical Principles for the Engineering Profession', which titled "Respect for life, law, the environment and public good", with comments on possible application for BCI products.

\section{Ethical Issues Regarding BCI Technologies}

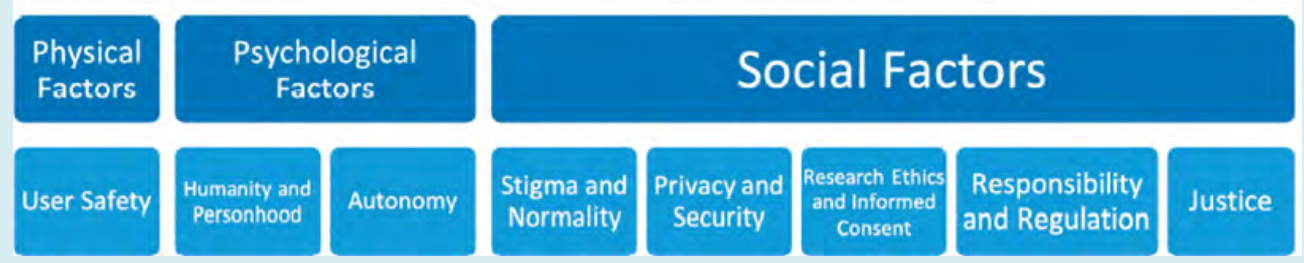

Figure 3: A summarizing show which covers all possible ethical issues in BCI that proposed by Coin A, et al. [9].

As it has been mentioned, there are six groups of ethics challenges of BCI Neuralink's devices. The first one is the device has to be lawful and justified work, which means that the device has to be approved before its ethics issues will be considered. For example, Neurohype, which means publicity for some devices and technologies before they have been approved by scientist society, or even before they have been made, which are technologies false and exaggerated or even unsubstantiated claims Dadia T, et al. [10]. The second challenge is to clarify the hazards and research ethics officially from the producer and the organizations. Where, spreading the culture and increasing the awareness regarding the $\mathrm{BCI}$ devices not just between the specialist scientist but also between public society, such as the EU and the UNESCO, play a significant role to solve the fair access of both technology and its ethics [5]. Therefore, the transparency of using these devices has to be highly clear to avoid the deception with or without intention, and to make and encourage equality and fair in the society. The third issue is the physical safety. As Neuralink's devices and technology involves inserting thousands of electrodes into the brain surface, it uses by helping a robot to identify and avoid targeting neurons and blood vessels as possible [9]. However, this process may produce physical safety issues such as infections and distortions of neurons, tissues and blood vessels, or reactions of the immune system. Therefore, the developing and improving in these robot technologies are necessary to minimize the physical safety concerns.

Another point is the privacy that is related to personal information, and identity issues. As this device uses a USB interface, $\mathrm{Wi}$-Fi or Bluetooth ways to transmit the data, which are high likely to be hackered with or without permission, and as an implanted device that gives physical painful under some conditions as it has been mentioned before, together raising hybrid ethical concerns [10]. This could be a big issue in terms of safety consideration. As a result of that, the scientists and suppliers community should provide high safety that the access for these devices only under control of the users. For example if a person have done a crime and consequently the police wanted to access to their device to do the investigation [11]. This police procedure may produce high ethics concerns of the personality data and privacy, which could be widely dangerous and harmful in nondemocracy governments. Kellmeyer P, et al. [12] mentioned that the data protection of the brain have to be in the top of safety guidelines and protection regulations in BCI devices, to solve the important ethical issue in this technology that is personal data protection and mental privacy of users [12]. People should have high ability, freedom and right to protect their private neural and mental information Yuste R, et al. [8]. Also, it is clear that the data will be one of the main 
things that sell in what called the black market, which needs to stablish new laws to organize and control that.

The agency, autonomy, and responsibility play significant ethical concerns in BCI devices uses. This ethics issue could be happened by shared such a hybrid agency between these implanted devices and human body that makes limitation in the humanity autonomy range in different activities of life. Kellmeyer P, et al. [5] pointed out implanting implanted devices inside humanity brain giving a permission and high ability of these systems in some cases to share the brain thinking or processing activities and maybe making decisions [5]. That is raising a question whether a human or a machine is who would be responsible for machine errors if it happened, which makes un-follow of public rules or leads to an unintended accident.

Additionally, the bias, justice, and normality challenges have high impact on the application of these technologies. As these devices have been manufactured and engineered by a human, to follow a specific direction of brain activity to distinguish based on for example gender, ethnicity, or colour, which maybe produce interaction bias of planted brain. Consequently, these criteria could encourage more differences between society levels and its culture that makes the community is in more unstable situation [13]. There are different possible ways to deal with this challenge by computer and data scientists under study Courtland R, et al. [14]. Also, the possible ethical concern with increasing in intelligence and memory of specific and selected human groups with that hidden device [10]. As a result for that, the device has to be obvious, and not hidden for the society, by setting rules for that. Regarding the equality of access issues that encourage inequalities in the society, these devices have to be available to all people those need it, which may apply by new technologies to make it cheaper or by establish laws to in force the companies [15].

\section{Recommendations}

There are different areas that could be used to deal with these mentioned the six ethics issues, such as establishing the regulations, rules, and laws depending on the public ethnicities, religious and socio-economic culture for people. Another way by enhancing and improving the technology ability or by mixing technology and laws together depending on the conditions of a situation. For example: creating high restricted laws that prevent and minimize technologies false and exaggerated or even unsubstantiated claims, to define the lawful and justified work. Also, establishing high transparency laws of using these devices to avoid the deception with or without intention, and to make and encourage equality and fair in the society.
Additionally, developing and improving in surgery robots technologies are necessary to minimize the physical safety concerns in the inserting and establishing process of $\mathrm{BCI}$ devices inside the brain. Also, the scientists and suppliers community should provide safe technologies that the access for these devices only under control of the users. Also, establishing laws that protect the personal data from using by others, and organize data marketing and transmitting if that will be possible to solve the related issues with the privacy, personal information, and identity.

The agency, autonomy, and responsibility issues have become a significant challenge, which could solve by making high restricted of laws and developing these device technologies to make a clear limit between human brain and the BCI activities. It is supposed that setting rules and developing new cheap technologies are both important factors to deal with the bias, justice, and normality challenges of these devices contain BCI. For example, new technologies with cheap price could make these devices available to all people whom need it, which is making the equality of access to these devices, to solve inequalities issues in the society. The restricted laws, which apply to force people who are using these devices to show it in a transparency way, and it has to be obvious for other people, might solve the related issues of the bias and makes it more justice.

\section{References}

1. Fourneret É (2020) The Hybridization of the Human with Brain Implants: The Neuralink Project. Camb Q Healthc Ethics 29(4): 668-672.

2. (2020) Neuralink Progress. Neuralink.

3. Collinger JL, Wodlinger B, Downey JE, Wang W, Tyler-Kabara EC, et al. (2013) High-performance neuroprosthetic control by an individual with tetraplegia. The Lancet 381(9866): 557-564.

4. Rainey S, Maslen H, Mégevand P, Arnal LH, Fourneret E, et al. (2019) Neuroprosthetic speech: The ethical significance of accuracy, control and pragmatics. Camb Q Healthc Ethics 28(4): 657-670.

5. Kellmeyer P (2019) Artificial intelligence in basic and clinical neuroscience: opportunities and ethical challenges. Neuroforum 25(4): 241-250.

6. Steinert S, Friedrich O (2020) Wired emotions: Ethical issues of affective brain-computer interfaces. Science and engineering ethics 26(1): 351-367.

7. Musk E (2019) An integrated brain-machine interface platform with thousands of channels. JMIR Publication 
21(10): e16194.

8. Yuste R, Goering S, Bi G, Carmena JM, Carter A, et al. (2017) Four ethical priorities for neurotechnologies and AI. Nature News 551(7679): 159.

9. Coin A, Mulder M, Dubljević V (2020) Ethical Aspects of BCI Technology: What Is the State of the Art? Philosophies 5(4): 31.

10. Dadia T, Greenbaum D (2019) Neuralink: The Ethical 'Rithmatic of Reading and Writing to the Brain. AJOB neuroscience 10(4): 187-189.

11. Weinberger S, Greenbaum D (2016) Are BMI prosthetics uncontrollable Frankensteinian monsters?. BrainComputer Interfaces 3(3): 149-155.
12. Kellmeyer P (2018) Big brain data: On the responsible use of brain data from clinical and consumer-directed neurotechnological devices. Neuroethics pp: 1-16.

13. Knight W (2017) Biased Algorithms Are Everywhere, and No One Seems to Care.

14. Courtland R (2018) Bias detectives: the researchers striving to make algorithms fair. Nature 558(7710): 357357.

15. Inglesant $P$, Hartswood M, Jirotka, M (2016) Thinking ahead to a world with quantum computers. The landscape of responsible research and innovation in quantum computing. 